\title{
Expression of viral and cellular cycle proteins and proteinases in cervical carcinoma cell lines as possible immunocytochemical markers of malignant phenotype
} Salvador Vázquez-Vega ${ }^{1}$, Patricia Sánchez-Suárez ${ }^{1}$, Alejandro GarcíaCarrancá $^{2}$ and Luis Benítez-Bribiesca*1

Address: ${ }^{1}$ Unidad de Investigación Médica en Enfermedades Oncológicas, IMSS, Mexico and ${ }^{2}$ Laboratorio de Virus y Cáncer, INCAN, Meixo Email: Luis Benítez-Bribiesca* - luisbenbri@mexis.com

* Corresponding author

from $24^{\text {th }}$ Annual Meeting of the National Cancer Institute of Mexico Mexico City, Mexico. I4-17 February 2007

Published: 5 February 2007

BMC Cancer 2007, 7(SuppI I):A2I doi:I0.I I86/I47I-2407-7-SI-A2I

This article is available from: http://www.biomedcentral.com/I47I-2407/7/SI/A2I

(c) 2007 Vázquez-Vega et al; licensee BioMed Central Ltd.

\section{Background}

Cervical cytology has been used extensively as a screening procedure for cervical cancer. Recent molecular developments increase its sensitivity and sensibility including HPV assessment, and the use of molecular biomarkers such as p16 protein. The aim of this study was to explore the pattern of expression of proteins from HPV, cell cycle and proteinases in various cell lines to determine the best candidates to serve as cytological biomarkers of malignancy.

\section{Materials and methods}

The following cell lines were used: Hela (HPV-18), Caski (HPCV-16), Siha (HPV-16), C33-A (HPV-negative); MCF7 (Breast cancer) and SW480 (Colon cancer) as negative controls. Slides were prepared from cultured lines and incubated with the following primary antibodies: E6, E7, Rb, p53, p16 INK4A, p14ARF, E2F1, Cyclins A, E and B1, MMP2, 9, 10, 11 and 12; Cathepsins B, D and F; and PCNA and Ki-67. Immunoreactivity was evaluated after staining with the DAKO-peroxidase system recording its cellular localization and intensity.

\section{Results}

All HPV positive cervical cell lines showed an intense nuclear staining for p16, except in C33-A that stained in the cytoplasm. Expression of p14 was intensely positive in the nucleoli of cervical carcinoma cell lines with or without HPV. Expression of replication proteins PCNA and Ki-
67 was intensely positive in the nucleus of all cell lines. Proteases were found intensely positive in the cytoplasm of all cells except for MMP12. Cell cycle proteins A, B1, E and E2F1 varied in intensity and cell location. Viral proteins E6 and E7 were found in low intensity and percentage in HPV positive cells. Rb and p53 were detected in the cytoplasm or nucleus of HPV positive cells.

\section{Conclusion}

Expression of p16 in the nucleus and p14 in the nucleoli are the most constant histochemical biomarkers in malignant cervical cell lines. Replication proteins PCNA and Ki67 and proteinases Cathepsin B and MMP are good markers of the malignant phenotype in dependent of the presence of HPV. The use of these biomarkers in conjunction might improve the sensitivity and diagnostic accuracy of cervical cytology. 